\title{
Current status of ex-vivo liver resection and autologous liver transplantation for end-stage hepatic alveolar echinococcosis
}

\author{
Chong Yang, Hong-Ji Yang, Shao-Ping Deng, Yu Zhang \\ Clinical Immunology Translational Medicine Key Laboratory of Sichuan Province \& Organ Transplantation Center, Sichuan Provincial People's \\ Hospital, University of Electronic Science and Technology of China, Chengdu, China \\ Contributions: (I) Conception and design: C Yang, Y Zhang; (II) Administrative support: HJ Yang, SP Deng; (III) Provision of study materials or \\ patients: None; (IV) Collection and assembly of data: C Yang, HJ Yang; (V) Data analysis and interpretation: C Yang, Y Zhang, SP Deng; (VI) \\ Manuscript writing: All authors; (VII) Final approval of manuscript: All authors. \\ Correspondence to: Yu Zhang. Professor, Clinical Immunology Translational Medicine Key Laboratory of Sichuan Province \& Organ Transplantation \\ Center, Sichuan Provincial People's Hospital, University of Electronic Science and Technology of China, Chengdu 611731, China. \\ Email: 68532815@qq.com.
}

\begin{abstract}
Hepatic alveolar echinococcosis (HAE) is a cosmopolitan zoonosis distribute widely in the northern hemisphere with high estimated 10-year mortality. Radical resection combined with oral albendazole administration are the major methods for HAE treatment, whereas most patients delayed diagnosis and treatment because which was considered as benign disease. For the cases with end-stage HAE could not be treated through conventional hepatectomy, allograft liver transplantation (LT) was regarded as a life-saving technique previously. However, graft shortage, high recurrence rate and long-term immunosuppressive therapy limited its utilization. Since the ex-vivo liver resection and autotransplantation (ERAT) procedure was first used in treating for end-stage HAE in 2011, there are more than 120 HAE cases patients were reported treating in this method up to now. Comparing with LT, ERAT needs neither an organ donor nor long term immunosuppressive therapy, and provide preferable overall survival rates. Based on the conventional ERAT procedure, some modification such as auxiliary partial autologous LT were introduced in the high selected end-stage HAE patients presently. However, the standard procedures for ERAT including surgical details and perioperative management have not been established because of limited reported cases. Also, the present ERAT experience for end-stage HAE treatment are all summarizes by the Chinese surgeon groups. For summarizing the knowledge and experience details, we reviewed present opinions about ERAT for end-stage HAE patients, and presented the future perspectives about this topic in this manuscript. We aimed at discussing the feasibility, indications, preparation, technical details, and postoperative outcomes of ERAT for HAE patients.
\end{abstract}

Keywords: Ex-vivo liver resection and autotransplantation (ERAT); hepatic alveolar echinococcosis (HAE); liver transplantation (LT); vascular reconstruction

Submitted Jan 11, 2020. Accepted for publication May 29, 2020.

doi: $10.21037 /$ apm-20-184

View this article at: http://dx.doi.org/10.21037/apm-20-184

\section{Introduction}

Alveolar echinococcosis (AE) is a cosmopolitan zoonosis caused by Echinococcus multilocularis (1). Hepatic AE (HAE), the main type of AE disease, have a distribution in the northern hemisphere, especially in China, the Russian Federation, continental Europe countries and North
America such as Alaska (2). The untreated HAE patients were estimated have a 10 -year mortality rate of $94 \%$ (3). Up to now, radical resection combined with oral albendazole are the major methods for HAE treatment (4). However, only $35 \%$ of patients are eligible for conventional hepatectomy because of delaying diagnosis and HAE being considered as benign disease $(5,6)$. 
As "parasitic cancer", the $\mathrm{AE}$ lesions' infiltrative growth may involve multi intro-hepatic ducts including biliary ducts, hepatic veins (HVs) or portal vein (PV) branches, which caused difficulty for conventional resection. For the end-stage HAE cases, multiple methods have been applied to cope with this disease which could not be conventional resected. However, palliative surgery, including lesion reduction surgery or cholangial drainage, has been proved not beneficial for long-term survival (7-9). Allograft liver transplantation (LT) was considered as a life-saving technique for "unresectable" cases previously $(10,11)$, whereas the graft shortage, high recurrence rate and long-term immunosuppressive therapy limited the utilization in endstage HAE (12-15). Also, HAE patients could not obtain a high enough score to be prior on the transplant list in US (16).

Based on the technique and experience in liver donor LT (LDLT), Wen et al. reported the first case showing that $e x$-vivo liver resection and autotransplantation (ERAT) was effective for end-stage HAE patient in 2011 (17). Comparing with allograft LT, ERAT requires neither an organ donor nor long term immunosuppressive therapy, and preferable overall survival rates (18). Up to now, more than 120 end-stage HAE cases have been treated by ERAT approach with ideal effect (19-21). Also, the application of artificial intelligence technique and new preserved solution study were also introduced into this area $(22,23)$. However, the standard procedures of ERAT for end-stage HAE have not been established because of limited reported cases.

Herein, we review the details and discuss the most recent advances of ERAT in the treatment of patients with endstage HAE, and aim at revealing the future direction of ERAT for this disease.

\section{Ex-vivo liver resection and autotransplantation}

ERAT approach was initially used in the advanced hepatic malignant tumors were deemed untreatable conventionally (24). For selected cases with main vascular structures were invaded, ERAT approach is opportunity for radical resection and long disease-free survival $(25,26)$. However, the outcome of ERAT technique for end-stage hepatic carcinoma is depressing because high incidence of unmanageable complications and postoperative recurrence (27-29). This reason is malignant tumor patients conventionally complicated with hypohepatia including cirrhosis and HBV or HCV infections, which may lead to more complication after this giant surgery. Also, the tumor stating is often too late in the selected cases for
ERAT approach, which may lead to rapid recurrence even the surgery completed. Then, this poor prognosis limited the application of ERAT for end-stage hepatic malignant tumors.

In contrast to malignant tumor, HAE is a relatively benign disease though it's tumor-like infiltrative growth, and the HAE patients combined with no hepatic hypohepatia conventionally. Also, the HAE lesions growth notable slowly compared with malignant tumor. This characteristic prompt the effect of ERAT technique in end-stage HAE is prior to it's in malignant hepatic tumors theoretically. To date, there are more than 120 end-stage HAE cases were treated using the ERAT approach (Table 1), and all the reported cases were initiated by Chinese surgeon group where bears $90 \%$ of the global burden of this disease.

\section{Candidate}

Because of the limited cases, there is no established guideline for ERAT in end-stage HAE patients. Generally, the selected cases for ERAT approach should be the HAE lesions could not be resected conventionally. As the infiltrative growth characteristics, the HAE lesions invaded intra-HVs needing long term for reconstruction. Also, the ERAT procedure should be considered if the giant lesions occupied the space for HVs reconstruction in situ. It should be indicated ERAT should be avoided if the patients with multi-organ $\mathrm{AE}$ which nether be removed nor controlled by albendazole administration.

The HAE lesion's "unresectable" using traditional techniques for end-stage HAE including: (I) the hepatocaval region including three HVs were invaded; (II) the retrohepatic vena cava (RHVC) was invaded; (III) the tertiary branches of the PV and portal arteries were invaded needing long time for reconstruction that ischemic time the liver couldn't tolerate $(17,19-21,33)$. Once the invaded intra- or extra-duct reconstruction could not complete in situ, an ERAT approach should be taken into account for radical HAE resection.

Based on the newly published expert consensus on diagnosis and treatment of HAE from China (35), the indications for ERAT included: (I) two or more hilars structure were invaded; (II) the invaded RHVC is more than $3 \mathrm{~cm}$ in length, or more than $180^{\circ}$ in circumference; (III) the invaded length of RHVC is less than $3 \mathrm{~cm}$, but infiltration above the confluence region of the HVs and below the diaphragm; (IV) obstructive jaundice, or severe first hilars invaded; (V) the ratio of RLV/body weight is 
Table 1 Indications and results of ERAT for end-stage HAE from 2011-2020 in PubMed

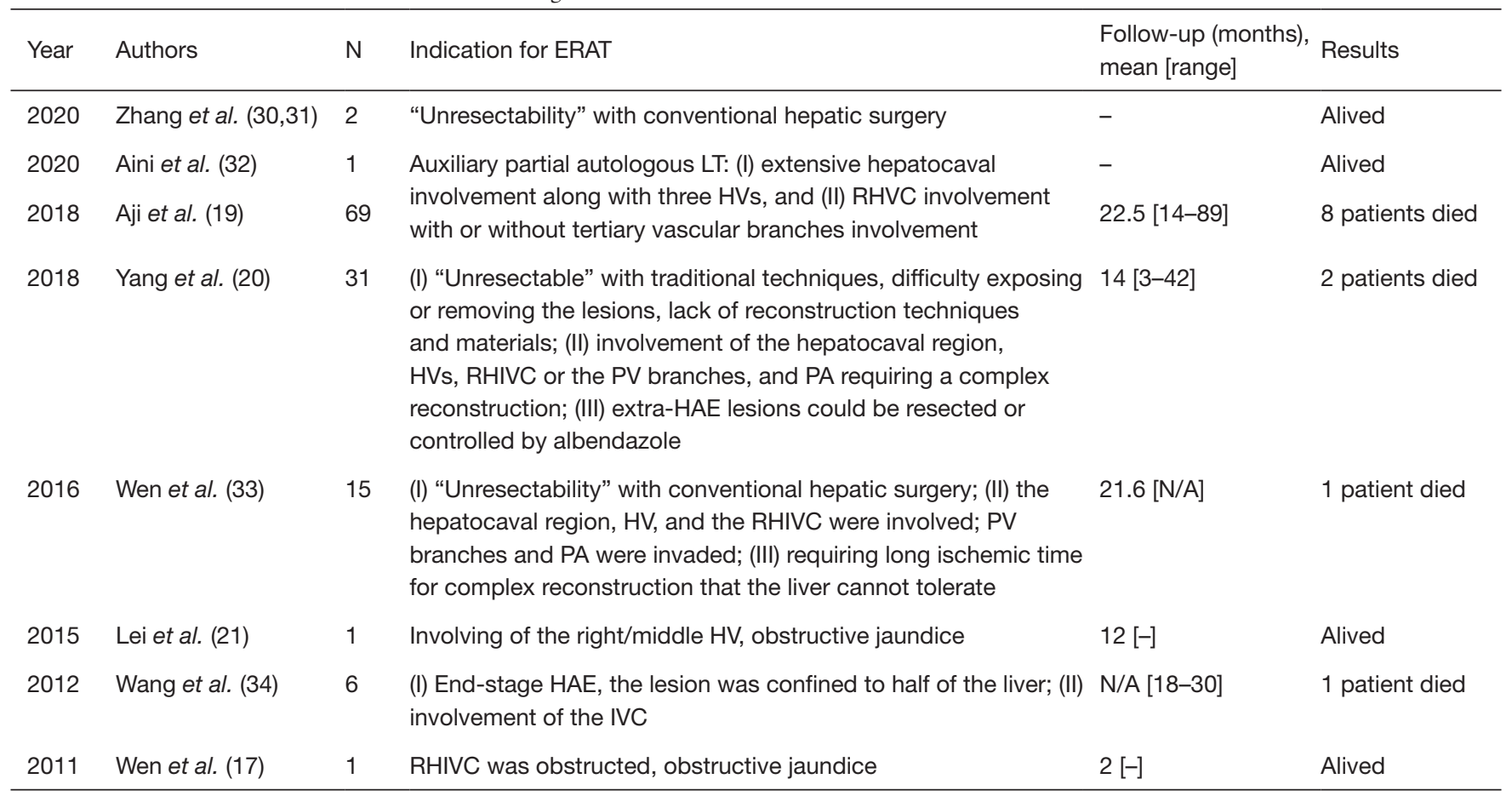

ERAT, ex-vivo liver resection and autotransplantation; HAE, hepatic alveolar echinococcosis; LT, liver transplantation; RHVC, retrohepatic vena cava; RHIVC, retrohepatic inferior vena cava; PV, portal vein; PA, portal arteries; HV, hepatic veins; IVC, inferior vena cava; N/A, data not available or cannot be counted.

more than $0.75 \%$.

At present, there are some end-stage HAE to be initiated ERAT approach previously could also be radical resected in situ with the development of surgical technology. Qiu et al. indicated type IV end-stage HAE patients with the opportunity to be operated in vivo based on "IHP" vascular infiltrated degree classification (36). For some cases, ante-situm liver resection with inferior vena cava (IVC) replacement procedure replaced ERAT procedure for radical resection for some special location $(28,37)$. And, the continuous pringle maneuver and in situ hypothermic perfusion through the inferior mesenteric vein (IMV) may resect the lesion in vivo, rather than removing the entire liver for lesion resection in vitro (38).

\section{Preoperative management}

Abdominal enhanced computed tomography (CT) and liver magnetic resonance imaging (MRI) is significant for surgical design and lesion volume/future liver remnant (FLR) calculation. Recent years, the application of threedimensional (3D) imaging analysis system is feasible to develop a reasonable scheme for liver resection and vascular anastomosis for end-stage HAE, which could effectively improve the success rate of liver ERAT and reduce the risks of surgery (39). Doppler ultrasound was needed to examine the greater saphenous veins' (GSV) length for potential vascular substitutes, and the digital subtraction angiography (DSA) was also needed to confirm the existence of tangible collateral circulation when total obliteration of RHVC was indicated by imaging studies (19).

The remnant liver volume (RLV) is a significant index for postoperative liver failure in ERAT (40). Based on the experience in LDLT, more than $40 \%$ of RLV/standard liver volume (SLV) is critical for the patients' prognosis (41). However, this index should be extended because there is no cirrhosis for HAE patients commonly. It is also accepted a minimum threshold of $25-30 \%$ of the SLV is enough for the functional demand during major resection $(42,43)$. Shen et al. revealed the ratio of RLV/SLV below $40 \%$ is safe for the end-stage HAE patients undergoing ERAT (44). Gruttadauria et al. indicated postoperative liver failure after hepatic resection for patients is highly occurred when the RLV less than $30 \%$ (45). Based on this 
index $(R L V=30 \%)$, Shen et al. made the surgical plan as whether resect the lesions in one stage or not for multiple giant HAE patients (46). Based on the author's experience, the RLV should be more than $30 \%$ for "health" liver before ERAT. If there is HBV or HCV infection, the index should be extended.

As the long germ growing period, the massive lesion may involve the blood supply (PV and/or HA branch) of the resection side, the procedure of hypertrophy for the contralateral hepatic parenchyma is similar to the effect of PV embolization (PVE) or partial associating liver partition and PV ligation for staged hepatectomy (ALPPS) $(47,48)$. Additionally, the HAE patients did not combine with hepatic disease commonly, which enables adequate compensatory function of the disease-free lobe. If the volume of diseasefree lobe is still small, the PVE procedure could also be considered aiming to increase the RLV and prevent postoperative hepatic failure (49). Recently, the ALPPS approach were also used in the radical resection for end-stage HAE patients with low RLV when PVE is unsuitable (50).

Some end-stage HAE patients combined with bile duct infiltrated causing obstructive jaundice. Obstructive jaundice or high level of serum bilirubin may critically impair the regenerative ability of the remnant liver (51), a routinely biliary drainage [percutaneous transhepatic cholangio drainage (PTCD) or endoscopic nasobiliary drainage (ENBD)] is needed to alleviate the high bilirubin index and biliary obstruction for an ideal Child-Pugh grade for ERAT. Yang et al. indicated the serum total bilirubin index should less than twice the upper limit of normal value before ERAT (20).

Obviously, serum bilirubin is not an isolated index for surgical indication, and some other situations should be taken into account. For example, HAE lesions invising the HVs may cause hepatic edema because of outflow obstruction. Preoperative percutaneous transhepatic HV stent implantation is an effective approach for alleviating hepatic edema and Budd-Chiari syndrome (52). This procedure could improve hepatic function obviously, and sequential ERAT approach may create radical resection opportunities for end-stage HAE patients (30).

\section{Surgical procedure}

In contrast to allograft LT, the ERAT procedure need long term for lesion resection and duct reconstruction during the anhepatic phase. An anhepatic phase for 1-1.5 hours without venous bypass is safe for the patient (53), whereas the term for HVs reconstruction is too long to tolerance. in the biggest series undergoing ERAT, the anhepatic phase is 360 [104-879] minutes (19). For most patients undergoing ERAT, a temporary IVC reconstruction and portosystemic shunt between the PV and hepatic IVC is needed to control hemodynamic stability during anhepatic phase.

A long anhepatic term may affect the hepatic venous reflux leading to internal environment disorder, intoxicants accumulation and hemodynamic changes (54). Shorten the anhepatic term is significant for reducing complications such as pulmonary thromboembolism and post-reperfusion syndrome (55). Based on the experience in LDLT $(56,57)$, the author's group modified the traditional ERAT procedure as resecting the lesions in vivo firstly, rather than remove the entire liver for lesion resection in vitro directly (31). Once excision plane reached the invaded HVs or RHIVC which could not be dissected from the HAE lesion in vivo, it's time for removing the liver into an ice bath for further bench resection. Up to now, Zhang group complete about 30 cases ERAT for end-stage HAE based on the modified procedure. This procedure could shorten the anhepatic term and cold ischemia phase. Also, if the encroached RHIVC range is short and easy to reconstruction in vivo based on detection, a halfway ex-vivo ERAT maybe enough for lesion resection, which could avoid complex ERAT procedure (34). And, this modified procedure may preserve more functional liver lobe(s) in situ (32).

Another reason for parenchymal transection in vivo firstly is to reduce potential bile leakage or bleeding after implantation. It's easier to confirm bleeding or bile leakage in the period of blood perfusion in vivo rather than $\mathrm{U}-\mathrm{W}$ or HTK solution perfusion in vitro, which could effectively reduce the blood loss after the autograft was replaced in situ. One inadequacy is which need to block the first hepatic portal frequently during parenchymal transection in vivo. Whereas the remnant liver is commonly disease free, which permit frequent first hepatic portal block without serious hepatic function influence.

The outflow reconstruction of the autologous graft is the surgical difficulty for ERAT. For avoiding graft rejection, autologous vein grafts should be the ideal material for vascular reconstruction. GSV, IMV and noninvaded IVC wall excided from AE lesions is the commonly used materials for outflow reconstruction $(10,58)$. Also, opened ligamentum teres hepatis can also be used as venous patch grafts in LDLT (59). When a long vascular was involved which could not be repaired using limit autologous vein grafts, an artificial vascular is need. The 
management of the anhepatic phase is also significant for patients' smooth recovery, a temporary IVC reconstruction should be performed regularly. Also, a temporary porto caval shunt through PV-artificial IVC anastomosis (end to side) were also needed for reducing intestinal congestion. Whether the permanent artificial IVC reconstruction is needed depends on the outflow of the collateral circulation. IVC reconstruction could be avoided in some special cases including vena azygos opening and rich collateral circulation established, non-lower extremity edema, and non-pressure difference between supra- and inferior hepatic vena cava (60-63).

On the other hand, peri-operative PV pressure (PVP) measurement is important for assessment of outflow patency. Ito et al. revealed that PVP $>20 \mathrm{mmHg}$ is strongly associated with poor survival after LDLT (64). Also, Ogura et al. indicated PVP $<15 \mathrm{mmHg}$ is a key for successful adult LDLT utilizing smaller grafts than before (65). Based on this experiment in LDLT, we prompt pre-operative RLV and peri-operative PVP measurement is an effective method to evaluate the liver reserve function. For the case with a high pre-operative PVP index whereas which drop down to a low level, it may indicate a fine prognosis. On the contrary, once the PVP didn't drop to an ideal level or even higher after the ERAT procedure finished, it may prompt poor prognosis caused by small-for-size syndrome, $\mathrm{PV}$ or reconstructed $\mathrm{HV}$ outflow obstruction.

\section{Post-operative management}

The post-operative management for ERAT is similarly to LT. As non allograft implanted, the immunosuppressive therapy isn't needed conventionally. Whereas the coldstored allograft vascular may using as materials for inflow (PV) and outflow (HVs, RHIVC) reconstruction may cause immune reaction similar to the process in solid organ transplantation $(66,67)$. Then, a low dose and shortterm immunosuppression therapy may be needed after allograft vascular transplantation for reducing thrombosis formation. Also, the artificial vascular implantation is a reason for thrombosis and even infection. The prophylactic anticoagulant and antibiotic therapy were needed for all patients after ERAT. Yang et al. (20) recommend the patients after ERAT should take warfarin sodium tablets for at least half 1 year refers to the international normalized ratio (INR) value is 2.0-3.0. Also, all patients should be administered albendazole $(15 \mathrm{mg} / \mathrm{kg} /$ day $)$ routinely for 1-2 years after ERAT $(68,69)$.
The most frequently postoperative complications after ERAT for HAE patients is pleural effusion as Aji et al. reported, which occurred in 18 cases of total 69 patients (19). The main reason for pleural effusion may be the long surgical time, or long time for RHIVC blocking during the anhepatic phase. Comparing with conventional hepatectomy and allograft LT, the special complication for ERAT is the outflow stenosis which may cause recurrent massive ascites (70). Once the HV-IVC anastomotic stenosis was suspected, the hetophlebography and tentatively stent implantation was needed to deal with the Budd-Chiari syndrome.

\section{Future perspectives}

To date, available data on ERAT for end-stage HAE have demonstrated therapeutic efficacy. As an alternative therapy to LT for end-staged HAE patients, ERAT procedure needed neither organ donor nor long-term immunosuppressive agents. However, there are limited cases reported and all the published papers about ERAT in HAE is from China up to now. More cases are needed for evaluating the effectiveness or safety and standardized surgical procedures establishment of ERAT for HAE. Also, the post-operative management procedure needed more cases treatment experience to standardization. For instance, the plan for immunosuppression therapy after allograft vascular used for vascular reconstruction (need or not? and plan) and albendazole administration ( 1 or 2 years?) is still controversial in different center. And, the experience from non-Asian country is need for future reported.

\section{Conclusions}

ERAT procedure is an effective and novel surgical method for end-stage HAE treatment. this method needs neither an organ donor nor long term immunosuppressive therapy and with preferable overall survival rates compared with allograft LT. However, the treated cases are limited up to now, more experience worldwide were need for the standardized management procedures establishment.

\section{Acknowledgments}

Funding: None.

\section{Footnote}

Conflicts of Interest: All authors have completed the 
ICMJE uniform disclosure form (available at http://dx.doi. org/10.21037/apm-20-184). The authors have no conflicts of interest to declare.

Ethical Statement: The authors are accountable for all aspects of the work in ensuring that questions related to the accuracy or integrity of any part of the work are appropriately investigated and resolved.

Open Access Statement: This is an Open Access article distributed in accordance with the Creative Commons Attribution-NonCommercial-NoDerivs 4.0 International License (CC BY-NC-ND 4.0), which permits the noncommercial replication and distribution of the article with the strict proviso that no changes or edits are made and the original work is properly cited (including links to both the formal publication through the relevant DOI and the license). See: https://creativecommons.org/licenses/by-nc-nd/4.0/.

\section{References}

1. McManus DP, Zhang W, Li J, et al. Lancet 2003;362:1295-304.

2. World Health Organization. Echinococcosis: epidemiology. Accessed on 14 Oct 2017. Available online: http://www.who.int/echinococcosis/epidemiology/en/

3. Feng X, Qi X, Yang L, et al. Human cystic and alveolar echinococcosis in the Tibet Autonomous Region (TAR), China. J Helminthol 2015;89:671-9.

4. Kern P, Wen H, Sato N, et al. WHO classification of alveolar echinococcosis: principles and application. Parasitol Int 2006;55 Suppl:S283-7.

5. Li F, Yang M, Li B, et al. Initial clinical results of orthotopic liver transplantation for hepatic alveolar echinococcosis. Liver Transpl 2007;13:924-6.

6. Bresson-Hadni S, Koch S, Miguet JP, et al. Indications and results of liver transplantation for Echinococcus alveolar infection: an overview. Langenbecks Arch Surg 2003;388:231-8.

7. Du C, Liu Z, Yang X, et al. Hepatectomy for patients with alveolar echinococcosis: long-term follow-up observations of 144 cases. Int J Surg 2016;35:147-52.

8. Buttenschoen K, Gruener B, Carli BD, et al. Palliative operation for the treatment of alveolar echinococcosis. Langenbecks Arch Surg 2009;394:199-204.

9. Kadry Z, Renner EC, Bachmann LM, et al. Evaluation of treatment and long-term follow-up in patients with hepatic alveolar echinococcosis. Br J Surg 2005;92:1110-6.
10. Aydinli B, Ozturk G, Arslan S, et al. Liver transplantation for alveolar echinococcosis in an endemic region. Liver Transpl 2015;21:1096-102.

11. Ozdemir F, Ince V, Barut B, et al. Living donor liver transplantation for Echinococcus alveolaris: single-center experience. Liver Transpl 2015;21:1091-5.

12. Pang C, Chu YK. Recurrence of liver transplantation combined with lung and diaphragm resection for alveolar echinococcosis: a case report. Transplant Proc 2015;47:2278-81.

13. Mantion GA, Vuitton DA. Auto-versus allo-transplantation of the liver for end-stage alveolar echinococcosis? Chin Med J (Engl) 2011;124:2803-5.

14. Koch S, Bresson-Hadni S, Miguet JP, et al. Experience of liver transplantation for incurable alveolar echinococcosis: a 45-case European collaborative report. Transplantation 2003;75:856-63.

15. Mosimann F, Bettschart V, Meuli R. Mediastinal recurrence of alveolar echinococcosis after liver transplantation. Liver Transpl 2003;9:97-8.

16. Hwang R, Liou P, Kato T. Ex vivo liver resection and autotransplantation: an emerging option in selected indications. J Hepatol 2018;69:1002-3.

17. Wen H, Dong JH, Zhang JH, et al. Ex vivo liver resection followed by autotransplantation for end-stage hepatic alveolar echinococcosis. Chin Med J (Engl) 2011;124:2813-7.

18. Shen S, Kong J, Qiu Y, et al. Ex vivo liver resection and autotransplantation versus allotransplantation for endstage hepatic alveolar echinococcosis. Int J Infect Dis 2019;79:87-93.

19. Aji T, Dong JH, Shao YM, et al. Ex vivo liver resection and autotransplantation as alternative to allotransplantation for end-stage hepatic alveolar echinococcosis. J Hepatol 2018;69:1037-46.

20. Yang X, Qiu Y, Huang B, et al. Novel techniques and preliminary results of ex vivo liver resection and autotransplantation for end-stage hepatic alveolar echinococcosis: a study of 31 cases. Am J Transplant 2018;18:1668-79.

21. Lei JY, Hao JC, Wang WT, et al. Ex vivo liver resection followed by autotransplantation to a patient with advanced alveolar echinococcosis with a replacement of the retrohepatic inferior vena cava using autogenous vein grafting: a case report and literature review. Medicine (Baltimore) 2015;94:e514.

22. He YB, Bai L, Jiang Y, et al. Application of a threeDimensional reconstruction technique in liver autotransplantation for end-stage hepatic alveolar 
echinococcosis. J Gastrointest Surg 2015;19:1457-65.

23. Apaer S, Tuxun T, Li T, et al. Compared efficacy of University of Wisconsin and histidine-tryptophanketoglutarate solutions in ex-situ liver resection and autotransplantation for end-stage hepatic alveolar echinococcosis patients. Hepatobiliary Pancreat Dis Int 2019;18:430-8.

24. Pichlmayr R, Grosse H, Hauss J, et al. Technique and preliminary results of extracorporeal liver surgery (bench procedure) and of surgery on the in situ perfused liver. Br J Surg 1990;77:21-6.

25. Vicente E, Quijano Y, Ielpo B, et al. Ex situ hepatectomy and liver autotransplantation for cholangiocarcinoma. Ann Surg Oncol 2017;24:3990.

26. Wen PH, Lin KH, Chen YL, et al. Extracorporeal hepatic resection and autotransplantation using temporary portocaval shunt provides an improved solution for conventionally unresectable HCC. Dig Dis Sci 2013;58:3637-40.

27. Oldhafer KJ, Lang H, Schlitt HJ, et al. Longterm experience after ex situ liver surgery. Surgery 2000;127:520-7.

28. Ye Q, Zeng C, Wang Y, et al. Long-term outcomes of ante-situm resection and auto-transplantation in conventionally unresectable hepatocellular carcinoma: a single-center experience. Ann Transplant 2018;23:81-8.

29. Ozsoy M, Ozsoy Z, Yilmaz S, et al. Ex situ liver resection and partial liver autotransplantation for advanced cholangiocarcinoma. Niger J Surg 2019;25:97-100.

30. Zhang Y, Xie P, Yang C, et al. Percutaneous stenting of left hepatic vein followed by Ex vivo Liver Resection and Autotransplantation in a patient with hepatic alveolar echinococcosis with Budd-Chiari syndrome. Int J Surg Case Rep 2020;68:251-6.

31. Zhang Y, Lai ECH, Yang C, et al. In situ reconstruction of vascular inflow/outflow to left lateral liver section, exvivo liver resection and autologous liver transplantation of remaining liver remnant for hepatic alveolar echinococcosis. Int J Surg Case Rep 2020;69:39-43.

32. Aini A, Shao YM, Shalayiadang $P$, et al. Auxiliary partial autologous liver transplantation for high-selective alveolar echinococcosis: a proof of concept. Transplantation 2020;104:e138-9.

33. Wen H, Dong JH, Zhang JH, et al. Ex vivo liver resection and autotransplantation for end-stage alveolar echinococcosis: a case series. Am J Transplant 2016;16:615-24.

34. Wang H, Liu Q, Wang Z, et al. Clinical outcomes of ex vivo liver resection and liver autotransplantation for hepatic alveolar echinococcosis. J Huazhong Univ Sci Technolog Med Sci 2012;32:598-600.

35. Sichuan Clinical Medical Research Center for on Hydatidosis. Expert consensus on diagnosis and treatment of complex hepatic alveolar echinococcosis (2020 edited). Chinese Journal of Bases and Clinics in General Surgery 2020;27:18-23.

36. Qiu Y, Yang X, Shen S, et al. Vascular infiltrationbased surgical planning in treating end-stage hepatic alveolar echinococcosis with ex vivo liver resection and autotransplantation. Surgery 2019;165:889-96.

37. Angelico R, Passariello A, Pilato M, et al. Ante situm liver resection with inferior vena cava replacement under hypothermic cardiopolmunary bypass for hepatoblastoma: report of a case and review of the literature. Int J Surg Case Rep 2017;37:90-6.

38. Lin YM, Chiang LW, Wang SH, et al. Resection of an intra-operative ruptured hepatocellular carcinoma with continuous pringle maneuver and in situ hypothermic perfusion through the inferior mesenteric vein: a case report. World J Surg Oncol 2013;11:2.

39. Xu TT, Sun YQ, Zhang Q, et al. Value of threedimensional visualization technology in preoperative evaluation of liver autotransplantation for end-stage hepatic alveolar echinococcosis. Zhongguo Xue Xi Chong Bing Fang Zhi Za Zhi 2018;30:646-51.

40. Schindl MJ, Redhead DN, Fearon KC, et al. The value of residual liver volume as a predictor of hepatic dysfunction and infection after major liver resection. Gut 2005;54:289-96.

41. Miller CM, Quintini C, Dhawan A, et al. The international liver transplantation society living donor liver transplant recipient guideline. Transplantation 2017;101:938-44.

42. Pak LM, Chakraborty J, Gonen M, et al. Quantitative imaging features and postoperative hepatic insufficiency: a multi-Institutional expanded cohort. J Am Coll Surg 2018;226:835-43.

43. Kim HJ, Kim CY, Park EK, et al. Volumetric analysis and indocyanine green retention rate at $15 \mathrm{~min}$ as predictors of post-hepatectomy liver failure. HPB (Oxford) 2015;17:159-67.

44. Shen S, Qiu Y, Yang X, et al. Remnant liver-to-standard liver volume ratio below $40 \%$ is safe in ex vivo liver resection and autotransplantation. J Gastrointest Surg 2019;23:1964-72.

45. Gruttadauria S, Vasta F, Minervini MI, et al. Significance of the effective remnant liver volume in major hepatectomies. Am Surg 2005;71:235-40.

46. Shen HD, Chen KF, Li B, et al. Two-stage hepatectomy 
for multiple giant alveolar echinococcosis. Medicine (Baltimore) 2017;96:e7819.

47. Wu X, Rao J, Zhou X, et al. Partial ALPPS versus complete ALPPS for staged hepatectomy. BMC Gastroenterol 2019;19:170.

48. Schlegel A, Lesurtel M, Melloul E, et al. ALPPS: from human to mice highlighting accelerated and novel mechanisms of liver regeneration. Ann Surg 2014;260:83946; discussion 846-7.

49. Narula N, Aloia TA. Portal vein embolization in extended liver resection. Langenbecks Arch Surg 2017;402:727-35.

50. Akbulut S, Cicek E, Kolu M, et al. Associating liver partition and portal vein ligation for staged hepatectomy for extensive alveolar echinococcosis: First case report in the literature. World J Gastrointest Surg 2018;10:1-5.

51. Oldhafer KJ, Lang H, Malago M, et al. Ex situ resection and resection of the in situ perfused liver: are there still indications? Chirurg 2001;72:131-7.

52. Vogel J, Gorich J, Kramme E, et al. Alveolar echinococcosis of the liver: percutaneous stent therapy in Budd-Chiari syndrome. Gut 1996;39:762-4.

53. Ma HL, Fan XT, Shi XJ, et al. Clinical study of treatment for end stage liver alveolar echinococcosis with ex-vivo liver resection and autologous liver transplantation. Chinese Journal of Transplantation (Electronic Version) 2017;11:1-4.

54. Huang HW, Lu HF, Chiang MH, et al. Hemodynamic changes during the anhepatic phase in pediatric patient with biliary atresia versus glycogen storage disease undergoing living donor liver transplantation. Transplant Proc 2012;44:473-5.

55. Hoffmann K, Weigand MA, Hillebrand N, et al. Is venovenous bypass still needed during liver transplantation? A review of the literature. Clin Transplant 2009;23:1-8.

56. Zhang Y, Wen T, Yan L, et al. Clinical significance of detailed preoperative evaluation on donors in right lobe living donor liver transplantation. Hepatogastroenterology 2008;55:1725-8.

57. Zhang Y, Wen T, Chen Z, et al. Following up of liver transplantation using dual left grafts from living donorsone case. Hepatogastroenterology 2008;55:235-6.

58. Roveda L, Zonta A, Staffieri F, et al. Experimental modified orthotopic piggy-back liver autotransplantation. Appl Radiat Isot 2009;67:S306-8.

59. Ikegami T, Wang H, Imai D, et al. Pathological analysis of opened round ligaments as venous patch grafts in living donor liver transplantation. Liver Transpl
2013;19:1245-51.

60. Cheng F, Yang ZY, Zeng J, et al. Anesthesia management of modified ex vivo liver resection and autotransplantation. Ann Transplant 2018;23:274-84.

61. Ikegami T, Shirabe K, Yoshiya S, et al. One-step reconstruction of the right inferior hepatic veins using auto-venous grafts in living-donor liver transplantation. Surg Today 2013;43:769-76.

62. Ikegami T, Soejima Y, Taketomi A, et al. Explanted portal vein grafts for middle hepatic vein tributaries in living-donor liver transplantation. Transplantation 2007;84:836-41.

63. Wu H, Yan LN, Li B, et al. Hepatic venous outflow reconstruction in right lobe graft without middle hepatic vein. Hepatol Res 2007;37:1044-51.

64. Ito T, Kiuchi T, Yamamoto H, et al. Changes in portal venous pressure in the early phase after living donor liver transplantation: pathogenesis and clinical implications. Transplantation 2003;75:1313-7.

65. Ogura Y, Hori T, El Moghazy WM, et al. Portal pressure $<15 \mathrm{mmHg}$ is a key for successful adult living donor liver transplantation utilizing smaller grafts than before. Liver Transpl 2010;16:718-28.

66. Mirelli M, Stella A, Faggioli GL, et al. Immune response following fresh arterial homograft replacement for aortoiliac graft infection. Eur J Vasc Endovasc Surg 1999;18:424-9.

67. Spunda R, Hruby J, Mericka P, et al. Immunosuppressive protocols with tacrolimus after cryopreserved aortal allotransplantation in rats. PLoS One 2018;13:e0201984.

68. Vuitton DA, Azizi A, Richou C, et al. Current interventional strategy for the treatment of hepatic alveolar echinococcosis. Expert Rev Anti Infect Ther 2016;14:1179-94.

69. Guidelines for treatment of cystic and alveolar echinococcosis in humans. WHO Informal Working Group on Echinococcosis. Bull World Health Organ 1996;74:231-42.

70. Yang X, Zeng XT, Wang WT. Recurrent massive ascites three months after liver autotransplantation. IDCases 2019; 17:e0583.

Cite this article as: Yang C, Yang HJ, Deng SP, Zhang Y. Current status of ex-vivo liver resection and autologous liver transplantation for end-stage hepatic alveolar echinococcosis. Ann Palliat Med 2020;9(4):2271-2278. doi: 10.21037/apm-20-184 